Historic, Archive Document

Do not assume content reflects current scientific knowledge, policies, or practices. 



\title{
Seeds of Woody and Herbaceous Plants 1
}

\author{
COLLECTED AND OFFERED BY
}

\section{PINEHURST NURSERIES, Pinehurst, N. C., U. S. A. , ?}

\author{
CABLE ADDRESS : NURSERIES, PINEHURST, N. C.
}

TERIS: Cash with orders or satisfactory references from unknown correspondents.

Seeds offered in this list will be shipped immediately after ripening or subject to special directions by purchaser.

NOTE.- We warrant all our seeds fresh of last crop and take every precaution to have them perfectly true to name. We give no warranty, express or implied, as to name, description or any other matter of any seeds we send ont. If the purchaser does not accept the seeds on these terms he must return them at once and the money which has been paid us for them will be refunded.

$\frac{1}{2} \mathrm{oz}$. at oz. rates; $\frac{1}{2} \mathrm{lb}$. at lb. rates. For larger quantities than those given here prices will be sent on application.

\section{Seeds of Woody Plants.}

\begin{tabular}{|c|c|c|}
\hline ALBIZZIA JULIBRISSIN-Mimosa & $\begin{array}{l}\text { LB. } \\
.65\end{array}$ & \\
\hline NDROMEDA ma & .60 & 5.00 \\
\hline nitida-Fetterbush & .60 & 5.00 \\
\hline ASTANEA PUMII & .50 & 4.50 \\
\hline .10 & .50 & 4.50 \\
\hline .15 & $\pi$ & 7.00 \\
\hline HAMIAECYPARIS & .75 & \\
\hline CLETHRA ALN & .85 & 7.50 \\
\hline LIFTONIA LIG & .75 & 6.00 \\
\hline .10 & .30 & 2.75 \\
\hline NA-Persimmon_ & .30 & 2.75 \\
\hline EUONYMUS AMERICANA__. & .75 & \\
\hline FO'THER & & \\
\hline ORDONIA la & & \\
\hline ILEX daboon - & 2.00 & \\
\hline “ GLABRA-Inkberry & .75 & 6.00 \\
\hline opaca-Holly & .40 & 3.50 \\
\hline “ VERTICILLATA _...... & .50 & 4.50 \\
\hline UGLANS $N$ & .10 & $* 1.5$ \\
\hline IRGINIANA-Red Cer & .50 & \\
\hline & 1.00 & \\
\hline
\end{tabular}

* Bushel.
OZ. LB. $10 L B$.

LAGERSTROEMIA INDICA-Crape Myrtle _._. .20

LIQUIDAMBAR STYRACIFLUA—Sweet Gum_ .25 1.50 12.00 LIRIODENDRON TULIPIFERA-Tulip Tree_- $.10 \quad .50 \quad 4.50$

MAGNOLIA glauca-Sweet Bay-_-_-_-_-_-_-_._.-.15 1.2511 .00 “6 GRANDIFLORA_. $10 \quad .70 \quad 6.50$

IIELIA AZEDERACH-Chinaberry--_-------_. $.10 \quad .60 \quad 3.00$ " " TIIBRACULIFERA

Texas Umbrella Tree .10 $\quad .60 \quad 5.00$

MYRICA CERIFERA-Bayberry _-_-_-_-_-_-. . 10 1.20 10.C0

NYSSA SYLTATICA--Tupelo_-_-_-_-____. $10 \quad .40 \quad 3.50$ OXYDENDRON ARBORELY_Sourwood____ $.10 \quad .60 \quad 5.00$ PA WLONIA IMIPERIALIS _-_._. 10

PINUS australis-(Palustris) Long-leaf Pine - -- .30 2.2520 .00

" MITIS_Short-leaved Pine-_-_--_-_-_-_- .30 2.25 20.00

“ TAEDA-Old Field Pine _-_____- . 302.2520 .00

PLATANUS OCCIDENTALIS_Sycamore____- . 10 .30 2.75 PRUNUS CAROLINLANA - Carolina Mock-

Cherry $.10 \quad .60 \quad 5.00$

PYRLS ARBUTIFOLLA-Chokeberry (Red berries)

QUERCUS alba- White Oak

" laurifolia-Water Oak _-

" " DARLINGTON _. . $25 * 4.00$

† Peck. 


\section{PINEHURST NURSERIES, Pinehurst, N. C.}

\section{WOODY PLANTS-CONTINUED.}

OZ. LB. 10 LB

QUERCUS NIGRA-Blackjack Oak_-__-___- $\quad .15 * 3.00$

" PRINOS MONTICOLA--Chestnut Oak .25 †1.25 QUERCUS VIRENS-Live Oak__ . $30 * 5.02$ SASSAFRAS OFFICINALE _-____ . .75 SIIILAX Iaurifolia_Evergreen China Brier_____.15 .75 $6.50^{\circ}$ (Black berries)

" walteri-Deciduous China Brier_____- $15 \quad .75 \quad 6.50$ (Red berries)

LLMLS AMERICANA-Elm____ . $15 \quad .75$

TIBURNUM NUDUM-Possum Haw______. $10 \quad .60 \quad 5.00$

" $\quad$ PRUNIFOLIUII-Black Haw____. $.15 \quad .75 \quad 6.50$

VITIS ROTUNDIFOLIA-Muscadine Grape____ .25 $1.00 \quad 9.00$

YCCCA ALOIFOLIA-Spanish Bayonet_-_-_-_. .30

“ FILAMENTOSA-Adam's Needle :__- .30 1.25

ZANTHORRHIZA APIIFOLIA-Yellow Root_- .25 1.00

\section{Seeds of Herbaceous Plants.}

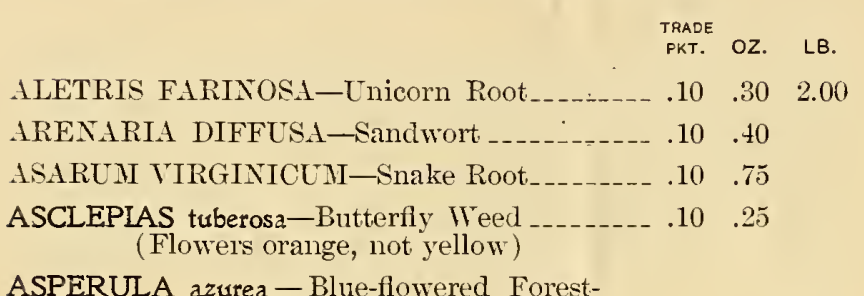

ASPERULA azurea-Blue-flowered Forest-

Master .10 .20 1.00

BAPTISIA TINCTORIA-False Indigo____ . 10 .20

BRETERIA PICKERINGII_-________. .20 1.00

CHINAPHILA MACULATA-Prince's Pine_-- . $10 \quad .40 \quad 2.00$

CHRYSOPSIS GRAMINIFOLIA-Scurvy Grass .10 .30

CLITORIA mariana-Butterfly Pea________._. $.20 \quad .50 \quad 5.00$

CIRSIUI REPANDUM-Scotch Thistle_-___- .10 $.20 \quad 1.00$

COREOPSIS AUREA-Golden Tickweed_-_-_. .20 .50

" LANCEOLATA___ $10 \quad .50$

" " $\quad$ ANGUSTIFOLIA $.10 \quad .50$

CROTALARIA SAGITTALIS-Rattlebox _....- . $.10 \quad .30$

CTENIUII AMERICANUM-Toothache Grass_ .10 .40 DATURA STRAMONIUM-Thorn Apple__.... . . 10 .25 2.25
DROSERA ROTLNDIFOLIA-Round-leaved

ERIOCALLON DECANGULAPE-Pipewort_-- .25 2.00 ERIOPHORUMI TIRGNICC M-Cotton Grass_. $.10 \quad .50$

GALACTIA glabella-Nilk Pea _______. $.10 \quad .20 \quad 1.50$ GERARDIA PURPUREA-False Fox Glove_-_ .10 .25 GNAPHALIUNI PLRPUREUM-Everlasting -- .10 $\quad .20 \quad 1.50$ IPONLAEA PANDERATA-Wild Potato Vine JATHROPHA stimulosa-Stinging Yettle --.--- .10 $\quad .30$ LIATRIS GRAMINIFOLIA-Button Snake Root .10 $\quad .20 \quad 2.00$

" SQUARROSA-Blazing Star____ .10 $\quad .20 \quad 2.00$

" TENUIFOLIA _

MIMULUS RINGENS-Monkey Flower--_-_- . 10 .50 OPUNTIA VULGARIS-Prickly Pear Cactus _- $.10 \quad .15 \quad .50$ LUPINUS diffusus-Deer Cabbage_-_______. $.10 \quad .40 \quad 3.60$ PASSIFLORA INCARNATA-Passion Flower _- .10 $.50 \quad 4.50$ PHLOX subulata-Moss Pink _-_-______- .25 2.00 RHEXIA MARIANA-Deer Grass_____.. $10 \quad .30 \quad 4.00$

" VIRGINICA-Yeadow Beauty_-___- $10 \quad .30 \quad 4.00$ RHYNCHOSIA ERECTA___ $.10 \quad .40 \quad 4.00$ “ RENIFORMIS SABBATIA brachiata_American Centaury____- .10 $.50 \quad 5.00$ SARRACENIA FLATA-Trumpet Leaf______- . $10 \quad .50 \quad 5.00$ "، $\quad$ PURPUREA_-Pitcher Plant___ .10 $.50 \quad 5.00$ " VARIOLARIS-Spotted

Trumpet Leaf $.10 \quad .50 \quad 5.00$

SCHRANKIA ANGUSTATA-Sensitive Plant_- .25 $\quad .60$ SERICOCARPUS CONYZOIDES - White-

Topped Aster .10 $\quad .30 \quad 3.00$

SILENE VIRGINICA--Fire Pink_-_-_-_-_-_.-. $10 \quad .25 \quad 2.00$ SISYRRHINCHIUM BERMUDIANTM-Blue-

Eyed Grass .10 .50

STILLINGIA SYLYATICA-Queen's Delight_. . $10 \quad .50 \quad 5.00$ TEPHROSIA V'IRGINICA-Devil's Shoe-String .10 $\quad .30 \quad 2.50$ TOFIELDIA PUBENS-Downy False Asphodel $.10 \quad .50 \quad 5.00$ TRADESCANTIA ROSEA-Rose Spiderwort_-_ .10 $\quad .70 \quad 7.00$ VERBASCUM THAPSES-Common Mullein_- .10 $\quad .20 \quad 1.00$ XYRIS BRETIFOLIA-Y̌llow-Eyed Grass____.

" CAROLINIANA _-_ $10 \quad .50 \quad 5.00$

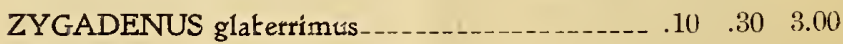


PINEHURST NURSERIES, Pinehurst, N. C.

\section{A Few Select Herbaceous Plants.}

(Prices for Plants are NET.)

\begin{tabular}{|c|c|c|}
\hline 10 & 100 & 1000 \\
\hline ALETRIS FARINOSA & 1.50 & 10.00 \\
\hline ASCLEPIAS TLBEROSA__ & 4.00 & 25.00 \\
\hline CHIMAPHILA MACULATA. & 3.00 & 20.00 \\
\hline YHRYSOPSIS GRAMINIFOLIA & 4.00 & \\
\hline CLITORIA MARIANA_. & 5.00 & 25.00 \\
\hline CIRSIUAI REPANDUML & 3.00 & \\
\hline COREOPSIS AUREA & 4.00 & \\
\hline LANCEOLATA & 2.50 & 15.00 \\
\hline ANGUSTIFOLIA_1.00 & 4.00 & \\
\hline CROTALARIA SAGITTALIS_ & 4.00 & \\
\hline CTENIUM AMERICANCM_- & 3.00 & 25.00 \\
\hline DIONAEA MUSCIPULA___-_. & 3.00 & 25.00 \\
\hline DROSERA ROTUNDIFOLIA_- & 2.00 & \\
\hline EPIGAEA REPENS & 3.00 & 20.00 \\
\hline ERIOCAULON DECANGULARE & 3.00 & 20.00 \\
\hline ERIOPHORUM VIRGINICUM & 4.00 & \\
\hline GALACTIA GLABELLA__-_. & 3.00 & 15.00 \\
\hline GENTIANA SAPONARIA _-- & 4.00 & \\
\hline HABENARIA CILIARIS & 3.00 & 20.00 \\
\hline HELCHERA AMERICANA_- & 6.00 & \\
\hline JATROPHA STIMULOSA _... & 3.00 & 15.00 \\
\hline LIATRIS GRAMINIFOLIA_- & 2.00 & 12.0 \\
\hline
\end{tabular}

\begin{tabular}{|c|c|c|}
\hline 10 & 100 & 1000 \\
\hline LITRIS SQLARROSA & 2.00 & 10.00 \\
\hline “ TENUIFOLL & 2.00 & 10.00 \\
\hline OPUNTIA VULGARIS___._- & 2.00 & 10.00 \\
\hline ORONTIUAI AGUATICL I_. & 4.00 & \\
\hline OSMUNDA CINNAMIOMEA & 4.00 & 25.01 \\
\hline PASSIFLORA INCARNATA & 3.00 & \\
\hline HLOX SUBULATA & 3.00 & 20.00 \\
\hline POLYGALA LUTEA & 2.50 & $10 . \mathrm{Cl}$ \\
\hline PYXIDANTHERA BARBLLATA & 4.00 & \\
\hline RHEXLA MARLANA & 3.00 & 15.00 \\
\hline VIRGIXICA & 2.50 & 10.00 \\
\hline RHYNCHOSIA ERECTA & 3.00 & 12.00 \\
\hline " RENIFORMIS & 2.00 & 10.00 \\
\hline SAGITTARLA VAR & 3.00 & \\
\hline SARRACENIA FLAYA & 4.00 & 25.00 \\
\hline " $\quad$ PLRPLREA & 3.00 & 20.00 \\
\hline VARIOLARIS & 4.00 & 20.0 \\
\hline SCHRANKIA ANGUSTATA ... & 4.00 & \\
\hline SILENE VIRGINICA _-_._. & 3.00 & \\
\hline SISYRRHINCHILM BERMLDLANUMI_-- .75 & 2.50 & 12.00 \\
\hline TEPHROSIA TIRGINICA _... & 4.00 & 25.00 \\
\hline TRADESCANTIA ROSEA & 3.00 & 20.00 \\
\hline XYRIS BREVIFOLIA _...... & 3.00 & 20.00 \\
\hline " CAROLINLANA__-_ & 2.00 & 10.00 \\
\hline ZYGADENLS GLABERRIMCS & 4.00 & 20.0 \\
\hline
\end{tabular}

Our new Wholesale Price List of Wcody and Herbaceous Plants for Fall 1898, will be ready by Septem. ber 1st. We shall be glad, however, to quote now, upon application, prices for any plants named in this list and for many others, of which we have no seed to offer this year. Herbaceous plants cau be shipped during nearly the entire summer with safety.

Our plants of Pinenurst Vior.et look as fine as ever. It is the hardiest form of the English Violet. Flowers are large, single, long-stemmed, very fragrant and of brilliant shade. It blooms continuously all winter in cold frame or open ground, has dark green foliage and is free of any disease. We offer clumps at 10 cents each, 75 cents per 10, $\$ 3.00$ per 100, $\$ 25.00$ per 1000. We shall be pleased to book your order now.

We solicit your correspondence about collecting native trees, shrubs and vines. It is one of our special ties and we take up many thousands of plants annually. 
